Kleczkowski, A. \& Kleczkowski, J. (1951). J. gen. Microbiol. 5, 346-356.

\title{
The Ability of Single Phage Particles to Form Plaques and to Multiply in Liquid Cultures
}

\author{
By A. KLECZKOWSKI AND J. KLECZKOWSKI \\ Rothamsted Experimental Station, Harpenden, Hertfordshire
}

SUMMARY: The results of testing a bacteriophage to a strain of clover nodule bacteria using young ( 1 day) and old ( 5 days) bacterial cultures both fit to the hypothesis that phage multiplication can be initiated by single phage particles. As the same phage preparations gave more plaques on solid media and higher proportions of liquid cultures in which phage multiplication could be detected, with young than with old bacterial cultures, the fit to the hypothesis is not an evidence that every single phage particle will multiply. It may be so when young bacterial cultures are used, although there is no positive evidence for it. With older bacterial cultures definitely only a proportion of viable phage particles succeed in starting phage multiplication, the proportion decreasing with the increasing age of bacterial cultures used for testing.

The two methods mainly used for quantitative estimation of bacteriophage are plaque counts and serial dilution in liquid media. These methods are satisfactory for estimating relative phage activities in different preparations of the same phage, but they are also used to estimate absolute numbers of phage particles. The translation of results obtained by either of the two methods into numbers of phage particles is based on the assumption, originally made by d'Herelle $(1917,1920,1926)$, that a single phage particle placed in a medium containing susceptible bacteria always causes general clearing in a liquid medium or local clearing (a plaque) on an agar medium. This assumption is often taken as selfevident, although observations have been recorded that make it doubtful.

Bronfenbrenner \& Korb (1925), who worked with a phage to 'Bacillus dysenteriae', found that estimates of numbers of phage particles by the plaque count were below those obtained by the dilution method. Similarly, Ellis \& Delbrück (1939) found that the estimates of numbers of a coli phage from plaque counts were about $40 \%$ of those estimated from the proportion of liquid cultures with demonstrable phage multiplication. Bronfenbrenner \& Korb (1925) also found that factors such as concentration of agar and the age of bacterial culture influence numbers of plaques. Clark (1927) showed mathematically that the assumption of a single phage particle being always sufficient to cause lysis in a liquid bacterial culture is not compatible with observed average proportion of parallel titrations giving the same dilution end-point.

Most workers translate the numbers of plaques directly into numbers of phage particles. Those who doubted the validity of this procedure, judged its efficiency by comparing the results with those obtained from liquid cultures, which were assumed to give a correct estimate of the numbers of phage particles, although this point was not established. The work described in this paper was done to test the value of both methods. Experiments were so designed that the results could be examined statistically for their compatibility 
with the assumption that single phage particles initiate multiplication in liquid cultures, irrespective of whether it is followed by obvious lysis of the culture, and could be compared with those obtained by the plaque-count method.

\section{MATERIALS AND METHODS}

Experimental. Clover nodule bacteria (Rhizobium trifolii, strain 317) were used as a host and the bacteriophage was obtained from soil as previously described (Kleczkowska, 1945) and isolated from a single plaque. Phage cultures referred to as 'stock cultures' were lysed liquid bacterial cultures passed through a Chamberland L3 filter. The media used were those used by Kleczkowska (1945).

Plaques were obtained by the poured plate method of Kleczkowska (1945). One volume of a phage preparation diluted in sterile liquid medium was mixed with nine volumes of a liquid bacterial culture, and $1 \mathrm{ml}$. of the mixture was mixed with $10 \mathrm{ml}$. of molten and cooled (to $42^{\circ}$ ) $0.7 \%$ agar medium, which was then poured into a Petri dish. The dilutions of phage preparations given in the text are the final dilutions in the liquid bacterial cultures, so that the dilution of a preparation is the fraction of $1 \mathrm{ml}$. of the preparation introduced into a plate. Plaques were counted after $48 \mathrm{hr}$. incubation at $25^{\circ}$.

The liquid bacterial cultures used for plating were obtained by inoculating a loopful from an agar culture into $9 \mathrm{ml}$. of sterile liquid medium, which was then incubated at $25^{\circ}$ for $\mathbf{2 4} \mathrm{hr}$. unless otherwise stated.

In experiments with liquid bacterial cultures, each tube containing $9 \mathrm{ml}$. of the culture was inoculated with $1 \mathrm{ml}$. of a phage preparation diluted in sterile liquid medium and $1 \mathrm{ml}$. of the sterile medium without phage was added to a control tube. The dilutions of phage preparations recorded are dilutions in the sterile medium, so that the dilution means the fraction of $1 \mathrm{ml}$. of an undiluted preparation inoculated into the bacterial culture.

The bacterial cultures used in any one experiment were prepared by adding a drop from a suspension to a series of tubes each containing $9 \mathrm{ml}$. of sterile medium. The tubes were incubated at $25^{\circ}$ for lengths of time given in the text and then inoculated with the phage. After such inoculation, the tubes were incubated for $24 \mathrm{hr}$. at $\mathbf{2 5}^{\circ}$ and then for 5 days at room temperature, during which time they were periodically inspected.

All the tubes that were not clearly lysed were tested for the presence of phage by placing a drop of their contents on the surface of an agar plate. Normal bacterial growth was taken as evidence that no phage was present. Two controls were run simultaneously with the test. In one a drop of the tested culture was placed on the surface of the agar plate and a drop of a phage culture added to it. The presence of bacterial growth here would mean either that a bacterial contamination of the culture had occurred or that a phageresistant bacterial variant had appeared. In the other control a drop of a normal culture of host bacteria was placed on the surface of the plate. This was done to provide a standard of comparison for normal bacterial growth. The method can be expected to detect phage only when present in such con- 
centrations that it is expected to occur in each drop, and when contamination with other bacteria has not occurred and no phage-resistant variant has appeared.

Statistical. The distribution of numbers of plaques obtained on different plates inoculated with the same dilution of a phage preparation is later shown to be Poissonian. Therefore, if $N$ is the number of plates and $M$ is the mean number of plaques per plate, $M / N$ is an estimate of the variance of the mean.

The probability $p_{i}$ of the phage occurring in a detectable quantity in a liquid bacterial culture inoculated with a given phage preparation at a dilution, will be shown to be

$$
p_{i}=1-e^{-z_{i} \lambda}
$$

The value of the constant $\lambda$ can be estimated by the method of maximum likelihood, and, consequently, the variance of the estimate can also be obtained.

Let $n$ be the number of liquid bacterial cultures inoculated with each of $m$ different dilutions of a phage preparation. Let $z_{1}, z_{2}, \ldots, z_{m}$ be the dilutions and $r_{1}, r_{2}, \ldots, r_{m}$, respectively, the number of cultures in which the presence of phage was detected. Putting $p_{i}=1-e^{-z i \lambda}$ and $q_{i}=e^{-z_{i} \lambda}$, the probability of obtaining the values of $r$ 's that were actually obtained would be

$$
\phi=\prod_{i=1}^{m}\left[\frac{n !}{r_{i} !\left(n-r_{i}\right) !} p_{i}^{r_{i}} q_{i}^{\left(n-r_{i}\right)}\right] .
$$

To obtain the estimate of $\lambda$ by the method of maximum likelihood, the value of $\lambda$ that makes the value of $\phi$ a maximum, must be chosen. This is done by solving for $\lambda$ the equation

$$
\frac{\partial}{\partial \lambda} \log \phi=\sum_{i=1}^{m}\left[\frac{r_{i}-n\left(1-e^{-z_{i} \lambda}\right)}{1-e^{-z_{i} \lambda}} z_{i}\right]=0 .
$$

The variance, $\sigma^{2}$, of the estimate of $\lambda$, if large samples are used, is then obtained from the equation

so that

$$
-\frac{1}{\sigma^{2}}=E\left(\frac{\partial^{2}}{\partial \lambda^{2}} \log \phi\right)=-E\left[\sum_{i=1}^{m} \frac{z_{i}^{2} r_{i} q_{i}}{p_{i}^{2}}\right]=-\sum_{i=1}^{m} \frac{z_{i}^{2} n q_{i}}{p_{i}}
$$

$$
\sigma^{2}=\frac{1}{n \sum_{i=1}^{m}\left[z_{i}^{2} e^{-z_{i} \lambda} /\left(1-e^{-z_{i} \lambda}\right)\right]} \text {. }
$$

If only one dilution, $z$, of a phage preparation is used, the equation (2) for obtaining the estimate of $\lambda$, becomes

$$
r / n=1-e^{-z \lambda},
$$

and the equation (3) for the variance becomes

$$
\sigma^{2}=\frac{1-e^{-z \lambda}}{z^{2} n e^{-z \lambda}}
$$

If estimates of $\lambda$ and also mean numbers of plaques per plate, are based on samples of reasonable size, they both can be considered to be approximately normally distributed estimates of some unknown existing values. As a variance 
can be assigned to any of these estimates, the standard deviation of a difference between any two estimates can be computed and the statistical level of significance of the difference assessed.

\section{RESUL'TS}

Inoculation of agar plates and liquid bacterial cultures with the same phage inoculum

Each of 200 agar plates and of 100 tubes containing a $24 \mathrm{hr}$. bacterial culture, was inoculated with $1 \mathrm{ml}$. of a phage stock culture diluted $10^{-9}$ in the $24 \mathrm{hr}$. liquid bacterial culture.

The mean number of plaques per plate was $\mathbf{1} \cdot \mathbf{6 5 5}$. Table 1 shows the observed distribution of plaque numbers on the plates and also the expected Poisson

\section{Table 1. The distribution of plaque numbers}

The mean number of plaques per plate:1.655.

\begin{tabular}{|c|c|c|c|}
\hline $\begin{array}{l}\text { No. of } \\
\text { plaques }\end{array}$ & $\begin{array}{l}\text { Observed } \\
\text { nos. of } \\
\text { plates }\end{array}$ & $\begin{array}{c}\text { Expected } \\
\text { nos. of } \\
\text { plates }\end{array}$ & $\begin{array}{c}\chi^{2} \\
\text { (D.F.=4) }\end{array}$ \\
\hline 0 & 45 & $38 \cdot 22$ & 1.2028 \\
\hline 1 & 58 & $63 \cdot 26$ & $0 \cdot 4374$ \\
\hline 2 & 51 & $52 \cdot 34$ & 0.0344 \\
\hline $\mathbf{3}$ & 28 & $28 \cdot 88$ & 0.0267 \\
\hline 4 & 10 & 11.94 & 0.3149 \\
\hline 5 & 3 ) & 3.96 & \\
\hline 6 & 3 & $1 \cdot 10$ & \\
\hline 7 & $2\} 8$ & \begin{tabular}{c|c}
$0 \cdot 26$ & $5 \cdot 36$
\end{tabular} & 1.3004 \\
\hline 8 & o & - & \\
\hline \multirow[t]{2}{*}{9} & 0) & -1 & \\
\hline & $\overline{200}$ & & $\overline{3 \cdot 3166}$ \\
\hline
\end{tabular}

distribution with the mean equal to $1 \cdot 655$. The value of the $\chi^{2}$ for the deviations of observed from expected numbers of plates shows that the deviations are not significant; (the value of $\boldsymbol{P}$ being about 0.5 ). It can be assumed, therefore, that the distribution of plaque numbers is Poissonian, as was found by Ellis \& Delbrück (1939) with a coli bacteriophage.

The Poissonian character of the distribution of plaque numbers can be considered as evidence that plaques are formed by single-phage particles. (Any aggregate of phage particles that is able to infect a bacterial cell as effectively as a single particle is considered as one particle.) However, only a proportion, say $\rho$, of particles able to form plaques may do so. If $\mu$ is the mean number of phage particles in $1 \mathrm{ml}$. of the inoculum, the mean number of plaques per plate would then be an estimate of $\rho \mu$.

Evidence is given below that multiplication of phage in a liquid bacterial culture can be initiated by a single phage particle. It is again possible that only a proportion, say $\rho^{\prime}$, of particles able to start multiplication, do so. If so, the proportion of cultures in which phage multiplication will be expected to occur, will be $p=1-e^{-\rho^{\prime} \mu}$. 
Among the 100 liquid cultures inoculated with the same inoculum as the agar plates, phage multiplication occurred in 85, all of which were lysed. Thus $0 \cdot 85=1-e^{-\rho^{\prime} \mu}$, so that $\rho^{\prime} \mu=1 \cdot 897$. It will be noticed that $\rho^{\prime} \mu$ corresponds to $\lambda$ of the equation (4) (with $z=1$ ), so that the variance of the estimate of $\rho^{\prime} \mu$ is given by the equation (5) and equals $0 \cdot 0567$.

Thus 1.655 is an estimate of $\rho \mu$ and 1.897 of $\rho^{\prime} \mu$, with variances 0.0083 and $\mathbf{0 . 0 5 6 7}$, respectively. The standard deviation of the difference between the two estimates is $\sqrt{(0 \cdot 0083+0 \cdot 0567)=0 \cdot 255}$. The ratio of the difference to its standard deviation, $\mathbf{0 \cdot 2 4 2} / \mathbf{0} \cdot \mathbf{2 5 5}=\mathbf{0 \cdot 9 5}$, corresponds to the value of $\boldsymbol{P}$ of about $0 \cdot 35$, so that the difference is not statistically significant. $\rho$ and $\rho^{\prime}$ may be equal, but their value is unknown. In other words, in the prevailing experimental conditions a similar proportion of phage particles both formed plaques and lysed liquid cultures. Every phage particle may have multiplied, but this is not established.

\section{Effect of age of bacterial culture on the numbers of plaques}

If only a proportion of phage particles form plaques, the proportion may vary with varying experimental conditions. To see whether age of bacterial culture influences the proportion, a phage stock culture was plated at a dilution of $10^{-7}$ with liquid bacterial cultures of three different ages: 1, 3 and 5 days. Ten plates were inoculated with the culture of each age. The mean numbers of plaques per plate were, respectively, $110 \cdot 9,98 \cdot 7$ and $88 \cdot 9$. Taking the variances of these means as equal to $1 / 10$ of their values, the difference between any two of the three means is statistically significant. Thus the older the bacterial culture the smaller was the proportion of phage particles that formed plaques. The size of plaques also decreased with the increased age of the bacterial culture. In all these respects the phage used in this work behaved similarly to a Shiga phage used by Bronfenbrenner \& Korb (1925).

\section{Relationship between phage concentrations and the proportions of liquid bacterial cultures with demonstrable phage multiplication}

Tables 2 and 3 show the results of four experiments, in each of which eight different dilutions of a phage preparation were made and 100 liquid bacterial cultures were inoculated with each dilution. The four experiments were made at different times using different phage-stock cultures. The stock cultures were diluted $10^{-8}$, and the values of $z_{i}$ given in the tables and in the text below show further dilutions of this. The tables record the numbers of cultures that were lysed and also the numbers of those that did not lyse but in which the presence of phage was detected by the method described above. The sums of the two numbers give the total numbers of cultures in which phage had multiplied. Unless the phage introduced in the inoculum had multiplied, its presence would be undetectable by the method.

In two experiments, 2-day, and in the other two experiments, 5-day bacterial cultures were used. The younger cultures were slightly opalescent and contained about $2 \times 10^{7}$ bacterial cells per ml.; the older cultures were strongly 
opalescent, contained about $2 \times 10^{8}$ bacterial cells per $\mathrm{ml}$. and some slimy sediment.

Simultaneously with each of the four experiments with liquid cultures, the phage stock cultures were also plated at a dilution $10^{-8}$ using $24 \mathrm{hr}$. bacterial cultures. The mean numbers of plaques per plate obtained on eight plates are given in the tables.

Let $\mu$ be the mean number of phage particles in $1 \mathrm{ml}$. of a phage stock culture diluted $10^{-8}$. If it be assumed that one phage particle can start phage multiplication in a liquid bacterial culture and that $\rho$ is the proportion of particles that will start multiplication in a given set of experimental conditions, the expected number of cultures in which the phage will multiply, would be

$$
Y_{i}=100\left(1-e^{-z i \rho \mu}\right)
$$

where $z_{i}$ is further dilution of the phage culture as defined above. $\rho \mu$ corresponds to $\lambda$ of the equations (1)-(3), so that the estimate of $\rho \mu$ by the method of maximum likelihood is obtained by solving the equation (2) for $\lambda$. If the assumptions on which the equation (6) is based are correct, the variance of the estimate of $\rho \mu$ will be given by the equation (3).

Tables 2 and 3 show the expected numbers of cultures in which phage had multiplied, computed by substitution into the equation (6) of the values of $\rho \mu$ obtained by the method of maximum likelihood. Tables 2 and 3 also show that for all the four experiments the values of $\chi^{2}$ obtained from deviations of observed from the expected numbers are not significant. The assumptions on which equation (6) is based, can, therefore, be assumed to be correct.

The values of the $\chi^{2}$ were computed according to the formula

$$
\chi^{2}=\Sigma\left(\frac{x_{i}^{2}}{Y_{i}}+\frac{x_{i}^{2}}{100-Y_{i}}\right)
$$

where $Y_{i}$ 's are the expected numbers of cultures where phage multiplication occurred, and $x_{i}$ 's are deviations of observed from the expected numbers. If, for a given phage dilution, the value of $Y_{i}$ or of $\left(100-Y_{i}\right)$ was smaller than about 5 , the values of $Y_{i}$, of $\left(100-Y_{i}\right)$ and of the corresponding observed numbers for two or more different phage dilutions were pooled, and the curly brackets in Tables 2 and 3 show where this was done. Each phage dilution, or group of pooled data, contributed one degree of freedom and there was a loss of one degree of freedom for the estimation of $\rho \mu$. Results obtained with some phage dilutions, with which all 100 cultures were expected to and did show phage growth, did not contribute to the estimation of $\rho \mu$ and were not used for computation of the $\chi^{2}$.

One purpose of the experiments shown in Tables 2 and 3 was to find if the value of $\rho$ varied with the age of bacterial cultures. This is not shown by comparing the values of $\rho \mu$ obtained in the experiments with the younger and the older cultures. The large scale of these experiments did not allow them to be run simultaneously, so that the value of $\mu$ could have varied from one experiment to another. However, the phage preparations used in each experiment were also tested on agar plates, using $24 \mathrm{hr}$. bacterial cultures for plating and keeping other experimental details as constant as possible. 


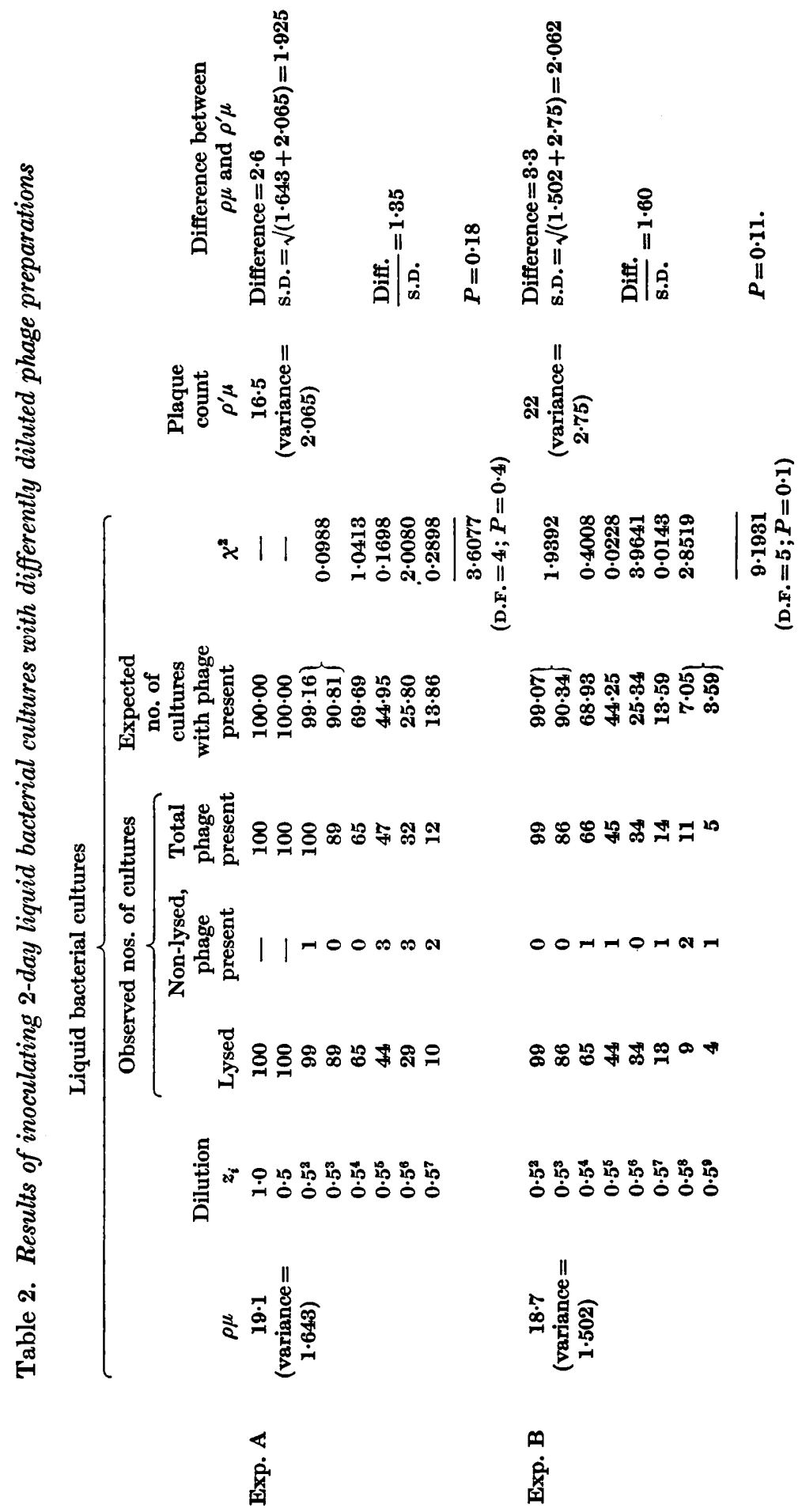




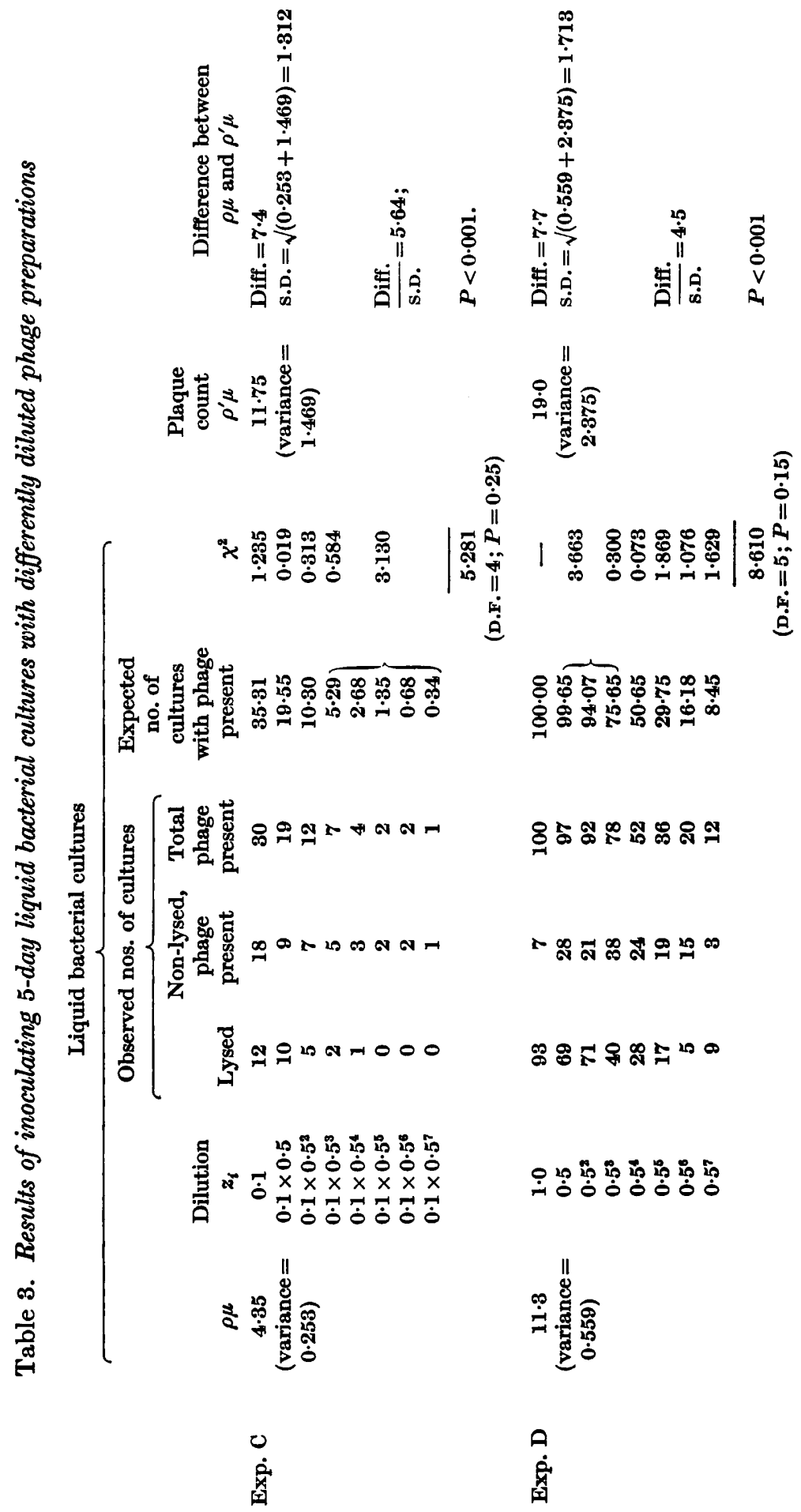


If $\rho^{\prime}$ is the proportion of phage particles that formed plaques, the mean number of plaques gives an estimate of $\rho^{\prime} \mu$, and the value of $\mu$, though unknown, is identical with that of $\mu$ of the corresponding experiment with the liquid cultures. The values of $\rho$ and $\rho^{\prime}$ can, therefore, be compared by comparing the estimates of $\rho \mu$ and $\rho^{\prime} \mu$.

It is not certain that the value of $\rho^{\prime}$ was constant in all tests, but it is assumed that it did not vary considerably. Thus the values of $\rho$ for liquid bacterial cultures of different ages can be compared indirectly by comparing them with the values of $\rho^{\prime}$.

Tables 2 and 3 give approximately statistical levels of significance of the differences between the estimates of $\rho \mu$ and of $\rho^{\prime} \mu$. The estimates of the values of $\rho \mu$ for the young bacterial cultures (Table 2 ) did not differ significantly from the estimates of $\rho^{\prime} \mu$. It is possible, therefore, that with the younger bacterial cultures $\rho=\rho^{\prime}$, and their value may have been about $1 \cdot 0$, but no evidence can be offered in favour of any particular value.

The estimates of the values of $\rho \mu$ for the older bacterial cultures (Table 3) were significantly smaller than the estimates of $\rho^{\prime} \mu$. For these cultures, therefore, $\rho<\rho^{\prime}$, so that the values of $\rho$ were definitely smaller than $1 \cdot 0$.

It is concluded that single phage particles can start multiplication in liquid bacterial cultures. When the cultures were 5 days old only a proportion of phage particles did this, and when younger cultures were used, the proportion was increased, but there is no evidence to show whether or not it reached 1.0 even under optimal conditions.

Tables 2 and 3 show that phage multiplication could occur without obvious lysis of the bacterial culture. This happened very rarely with the younger bacterial cultures (Table 2), but frequently with the older cultures (Table 3). The younger bacterial cultures became wholly clear when lysed, whereas the older cultures although obviously lysed were still somewhat opalescent.

\section{DISCUSSION}

The failure of some phage particles to multiply can have two possible meanings. If the phage population is homogeneous, each phage particle stands an equal chance of success, but the magnitude of the chance is determined by some conditions in the bacterial culture, such as heterogeneity of bacterial population, non-uniformity of bacterial surface or presence of inhibitory metabolic products in the medium. If the phage population is heterogeneous, a proportion of phage particles may be incapable of infecting, the magnitude of the proportion being determined by some conditions of the cell or of the surrounding medium. Experimental evidence of heterogeneity of phage populations, even after repeated isolations from single plaques, is supplied by the results obtained with a few coli phages by Andrewes \& Elford (1933), who found that phage particles differed in their reactions with antisera, and by Burnet \& Freeman (1937), who found that particles of a phage against Bacillus dysenteriae differed in their susceptibility to inactivating effect of a preparation obtained from an extract from host bacteria. 
If the behaviour of bacteriophages is analogous to that of animal and plant viruses, the results obtained with a bacteriophage might help to interpret the results obtained in the experiments designed to find whether one virus particle is sufficient to start a local infection in an animal or a plant, or whether a minimal dose containing many virus particles is necessary.

An equation equivalent to the equation (6) could not be fitted satisfactorily to numbers of symptoms of local infections with papilloma and vaccinia viruses (Bryan \& Beard, 1940) or with several plant viruses (Kleczkowski, 1950). The numbers could be fitted to the sigmoidal curve when logarithms of virus concentrations were plotted against proportions of inoculated susceptible regions of a host that developed local symptoms of infection. Bryan \& Beard (1940) concluded from this that the hypothesis of a single virus particle being able to form a local symptom is false, and that minimal effective virus doses vary as a result of variation in regional host susceptibility in such a way that logarithms of minimal effective virus doses are normally distributed. Kleczkowski (1950) considered Bryan \& Beard's conclusion as likely, but he drew attention to the fact that the hypothesis of single virus particles being able to form local lesions on plant leaves could be retained if an additional assumption were made that would make the value corresponding to $\rho$ of the equation (6) vary from one susceptible region to another, and a number of such possible assumptions was suggested. The results obtained with bacteriophage suggest a likely auxiliary assumption.

Let us assume that symptoms of local infections in animals and plants can be caused by single virus particles, and, by analogy with bacteriophage, let the auxiliary assumption be made that there is a limited probability that a virus particle, when placed in a susceptible region of host tissue, will successfully initiate a process of local infection. If susceptible regions are not identical, the magnitude of the probability may vary from one region to another, and, therefore, a value corresponding to $\rho$ of the equation (6) would not be constant within the same experiment, and this would account for the impossibility of fitting an equation similar to (6) to experimental results.

The equation (6) could be fitted to the results obtained when liquid bacterial cultures were inoculated with a variously diluted phage preparation, only because the value of $\rho$ was constant within one experiment, and this was so because all the bacterial cultures used within one experiment resembled one another closely. If variety were introduced into bacterial cultures, for example, by using in the same experiment bacterial cultures of different ages, the value of $\rho$ would not be constant but vary from one culture to another, and this would make it impossible to fit the equation (6) to experimental results. Therefore, the fact that an equation equivalent to (6) could not be fitted to experimental results obtained with some animal or plant viruses does not necessarily invalidate the hypothesis of one virus particle being able to initiate a local infection. 


\section{REFERENCES}

Andrewes, C. H. \& Elford, W. J. (1933). Observations on anti-phage sera. I. 'The Percentage Law.' Brit. J. exp. Path. 14, 367.

Bronfenbrenner, J. J. \& Korb, C. (1925). Studies on the bacteriophage of d'Herelle. III. Some of the factors determining the number and size of plaques of bacterial lysis on agar. J. exp. Med. 42, 483.

Bryan, W. R. \& Beard, J. W. (1940). Host influence on the characterization of response to the papilloma protein and to vaccinia virus. J. infect. Dis. 67, 5 .

Burnet, F. M. \& Freeman, M. (1937). A comparative study of the inactivation of a bacteriophage by immune serum and bacterial polysaccharide. Aust. J. exp. Biol. med. Sci. 15, 49.

Clark, H. (1927). On the titration of the bacteriophage and the particulate hypothesis. J. gen. Physiol. 11, 71 .

Ellis, E. L. \& Delrzứck, M. (1939). The growth of bacteriophage. J. gen. Physiol. $22,365$.

D'Hereme, F. (1917). Sur un microbe invisible antagoniste de bacilles dysentériques. C.R. Acad. Sci., Paris, 165, 373.

D'Herelle, F. (1920). Sur le microbe bactériophage. C.R. Soc. Biol., Paris, 83, 247.

D'Herelle, F. (1926). The Bacteriophage and its Behaviour. Translated by G. H. Smith; London: Baillière, Tindall and Cox; Baltimore: The Williams and Wilkins Co.

KLECZKOwSKA, J. (1945). The production of plaques by Rhizobium bacteriophage in poured plates and its value as a counting method. J. Bact. 50, 71 .

KLECZKOwsKI, A. (1950). Interpreting relationships between the concentrations of plant viruses and numbers of local lesions. J. gen. Microbiol. 4, 53. 